\title{
Factores limitantes del rendimiento en un 50 libre
}

\section{Álvaro Tomico Becerra \\ Universidad de Huelva Email: alvaro.tomico@alu.uhu.es}

\section{RESUMEN:}

El objetivo del presente trabajo es el de identificar los factores que determinan el rendimiento en un 50 libre, tarea que resulta fundamental para optimizar el entrenamiento de cualquier modalidad deportiva. Esta prueba, se constituye como la más corta, en cuanto a duración, de todas las que componen la natación competitiva, razón por la que la fuerza-explosiva o potencia -tanto en la salida como el en el nado juega un papel crucial en el éxito en dicha prueba. A su vez, la capacidad del deportista para mantener los valores óptimos de fuerzaexplosiva durante el transcurso de la prueba, se convierte en otro de los aspectos esenciales del rendimiento que va a repercutir en el tiempo final de la prueba.

A la hora de analizar los factores que influyen en el rendimiento de esta prueba, han sido excluidos aquellos relacionados con aspectos técnicos y biomecánicos, y se han tenido en cuenta los aspectos reglamentarios, las competiciones que realiza el deportista a lo largo de la temporada, la composición corporal idónea, las necesidades de fuerza específica, el perfil de resistencia aeróbico y el perfil de resistencia anaeróbico. Por último, se exponen algunos instrumentos para valorar el rendimiento del deportista a lo largo del proceso de entrenamiento, ya sea en la piscina o en el gimnasio.

PALABRAS CLAVE: Natación, 50 libre, rendimiento, fuerza-explosiva 


\section{INTRODUCCIÓN}

La natación competitiva se constituye como una modalidad deportiva realizada en el medio acuático, cuyo nivel de rendimiento se asocia a la capacidad del deportista de completar una distancia determinada en el menor tiempo posible (Willems, Cornelis, De Deurwaerder, Roelandt, y De Mits, 2014; Barbosa, Morais, Marques, Costa y Marinho, 2014). El hecho de que se desarrolle en el medio acuático, dota a este deporte de unas características muy peculiares. En primer lugar, porque se requiere un mayor gasto energético para desplazarse a través de ella, dado que el agua es aproximadamente 800 veces más densa que el aire (Caputo, Oliveira, Denadai, y Greco, 2006), y por tanto, el nadador tendrá que superar la resistencia que ésta ofrece para poder avanzar (Seifert et al. 2010). En segundo lugar, porque al estar inmerso en un medio inestable, tan solo una parte de la fuerza aplicada por el nadador se traduce en fuerza propulsiva (Caputo et al., 2006; Salo y Riewald, 2008), que es la que permite al nadador avanzar hacia delante. Por tanto, uno de los principales factores que determinan el éxito en este deporte se relaciona con la capacidad del deportista para maximizar la fuerza propulsiva y reducir la resistencia al avance que ofrece el medio acuático (Barbosa, Morais, Marques, Costa y Marinho; Caputo et al. 2006), lo que a su vez está muy relacionado, entre otros aspectos, con la habilidad técnica del individuo.

Las distintas pruebas que componen la natación competitiva van desde los 50 hasta los 1500 metros, y cada una de ellas se caracteriza por diferentes duraciones, intensidades (Figueredo, Zamparo, Sousa, Vilas-Boas, y Fernandes, 2011) y estilos natatorios, lo que supone que se produzcan distintas demandas en el organismo del nadador (Toussaint y Hollander, 1994), así como distintos requerimientos a nivel biomecánico.

En este trabajo se analizarán los diferentes factores que influyen en el rendimiento del 50 metros estilo libre realizado en piscina de 50 metros, tarea que resulta fundamental para optimizar el entrenamiento de cualquier modalidad deportiva. (Cajuela et al. 2007). La prueba de 50 metros libre suele realizarse al estilo crol, dado que es el más rápido de los cuatro estilos que componen la natación competitiva. Se constituye como la prueba más rápida, estando el récord del mundo masculino en 20,91 segundos en piscina de 50 metros, y en 20,30 segundos en piscina de 25 metros. Cuando se realiza en piscina de 50 metros, el tiempo total de la prueba está compuesto por el tiempo de salida (primeros 15 metros), tiempo de nado (desde los 15 a los 40 metros), y tiempo de llegada (desde los 40 a los 50 metros) (Arellano, Brown, Cappael y Nelson, 1994).

El rendimiento en la salida, es uno de los elementos claves en el rendimiento de esta prueba (West, Owen, Cunningham, Cook y Kilduff, 2011), pudiendo convertirse en hasta un $30 \%$ de la distancia total de la misma (Lyttle and Benjanuvatra, 2007; citado por Bishop, Smith, Smith y Rigby, 2009). La ejecución de una buena salida vendrá determinada por el tiempo de reacción, la potencia del salto y la habilidad para desplazarse a una alta velocidad durante el deslizamiento subacuático (Maglischo, 1993; citado por Breed y Young, 2003).

Por otro lado, la velocidad durante el nado, vendrá determinada en buena parte por la capacidad del nadador de generar fuerza máxima a una elevada frecuencia de brazada (número de brazadas por unidad de tiempo) (Girold, Maurin, Dugue, Chatard y Millet, 2007), de conseguir una óptima longitud de brazada (distancia recorrida por el nadador en cada brazada) (Girold, Jalab, Bernard, Carrete, Kemoun, y Dugue, 2012) y de mantener una correcta posición del cuerpo y una buena técnica (Monu, 2013) 
durante todo el transcurso de la prueba. En resumen, podemos decir que la velocidad máxima de nado, especialmente en distancias cortas, dependerá de la fuerza propulsiva generada por el nadador, así como de las capacidades técnicas y energéticas del mismo (Dopsaj, Matkovic y Zdravkovic, 2000).

Por último, el rendimiento en la llegada se verá influenciado por la capacidad del nadador para mantener los óptimos niveles de potencia y velocidad, así como la habilidad técnica durante los últimos 10 metros de la prueba, aunque evidentemente, lo segundo repercutirá sobre lo primero.

\section{Reglas que influyen en el rendimiento}

De entre las principales consideraciones que debemos tener presente a la hora de analizar de qué manera los aspectos reglamentarios influyen en el rendimiento de esta prueba, destacamos: 1) aquellas relacionadas con la forma con la que el nadador puede desplazarse por el agua y 2) aquellas que tienen que ver con la manera en la que el nadador inicia su puesta en acción, esto es, la salida.

Con respecto a la manera en la que el nadador puede desplazarse en un 50 libres, el reglamento de natación establece que en las pruebas de estilo libre, "el nadador puede nadar cualquier estilo, excepto en las pruebas de estilos individual o por equipos, en las que estilo libre significa cualquier estilo que no sea espalda, braza o mariposa". Dado que el reglamento no establece ninguna directriz sobre cómo nadar en estilo libre, el nadador podrá elegir la forma de nadar que sea más eficaz, es decir, con la que consiga completar la distancia en el menor tiempo posible. Pero aun así, es el estilo crol el que emplean la gran mayoría de los nadadores en las pruebas de estilo libre, puesto que es el estilo que se considera más rápido desde el punto de vista biomecánico (Troup, 1999; citado por Monu, 2013).

Por otro lado, se permite que durante los primeros 15 metros después de cada salida y cada viraje, el nadador pueda "permanecer completamente sumergido (...) En este punto (15 metros desde la pared) la cabeza debe haber roto la superficie". Esta norma puede favorecer la velocidad del nadador durante este tramo, puesto que al avanzar por debajo de la superficie del agua, el cuerpo del nadador no se vería expuesto a la resistencia que ofrece el agua en la superficie generada por el oleaje (Videler, 1993; citado por Arellano, Pardillo y Gavilán, 2002). Por tanto, siempre y cuando el nadador desarrolle una patada ondulatoria subacuática eficaz (Willems et al., 2014), el mantenerse por debajo de la superficie del agua durante la mayor distancia reglamentaria posible (15 metros desde la pared) contribuiría a disminuir el tiempo empleado en las fases de salida y viraje (Arellano, Pardillo y Gavilán, 2002), y con ello, el tiempo empleado en recorrer la distancia de la prueba.

Con respecto salida, decir que ésta se realizará desde fuera del agua, según la norma 4.1. del Reglamento de Natación 2013/2017 de la Real Federación Española de Natación, que establece que "la salida para las carreras de Estilo Libre, Braza, Mariposa y Estilos Individual, se efectuará por medio de un salto". El salto se realizará desde una plataforma situada en el borde de la piscina, y que se encuentra a una altura de entre 50 y 75 centímetros sobre el nivel del agua, después de que el Juez de Salidas dé la señal de salida.

Por este hecho, un tiempo de reacción rápido y una gran potencia de salto, son esenciales para un buen rendimiento en la salida (Breed y Young, 2003). Estos dos factores, unido a la capacidad del nadador para avanzar con rapidez mediante la patada ondulatoria subacuática durante los primeros 15 metros de la prueba, determinarán el rendimiento de la salida, que, como dijimos anteriormente, se 
convierte en un elemento clave del rendimiento en una prueba tan corta como es el 50 libre Factores limitantes del rendimiento en un 50 libre (Breed y Young, 2003; West et al. 2011; Rebutini, Pereira, Bohrer, Ugrinowitsch y Rodacki, 2014).

Las competiciones a las que se enfrenta el nadador a lo largo de la temporada pueden ser divididas en dos grupos: las competiciones de la temporada de invierno (en piscina de 25 metros) y las de verano (en piscina de 50 metros). Ambas determinan los dos macrociclos en los que se divide cada temporada, siendo el objetivo principal para el nadador llegar a su capacidad de rendimiento máxima en las competiciones más importantes de cada macrociclo (invierno y verano).

\section{COMPETICIONES ANUALES}

Las competiciones a las que se enfrenta el nadador a lo largo de la temporada pueden ser divididas en dos grupos: las competiciones de la temporada de invierno (en piscina de 25 metros) y las de verano (en piscina de 50 metros). Ambas determinan los dos macrociclos en los que se divide cada temporada, siendo el objetivo principal para el nadador llegar a su capacidad de rendimiento máxima en las competiciones más importantes de cada macrociclo (invierno y verano).

Según Vasconcelos (2000), podemos establecer tres tipos de competiciones, las cuales se corresponden con niveles de objetivos diferentes respecto a la participación del nadador. Estas son:

- Competiciones preparatorias: son aquellas en las que el nadador participa sin ningún tipo de preparación específica, incluyéndose en las etapas de preparación específica y precompetitiva, formando parte del propio entrenamiento. Tienen como principal objetivo, entre otros, la adaptación a las situaciones de competición, el desarrollo de una determinada cualidad motora o el refuerzo de la confianza en sí mismo.

- Competiciones importantes: estas competiciones representan un objetivo real para los nadadores y para el club. Desde el punto de vista psicológico, sirve para tomar contacto con las exigencias de las competiciones más importantes, dado que el nadador estará en disposición de hacer mejores tiempos debido a factores volitivos y a una preparación secundaria.

- Competiciones principales: estas competiciones representan el objetivo primordial de la temporada, en las que el nadador deberá alcanzar su pico máximo de forma. Para ello, se lleva a cabo una fase de preparación especial previa, denominada "tappering" o "puesta a punto", en la que se permite una recuperación total del organismo.

\section{COMPOSICIÓN CORPORAL}

Tanto la composición corporal como el somatotipo ideal varía en función de cada modalidad deportiva o de la prueba específica a la que se tenga que enfrentar el atleta (deGaray, Levine, Carter, 1974; citado por Siders, Lukaski y Bolonchuk, 1993). De hecho, Tanner (1964); citado por Camarero, Tella, Moreno, y Fuster (sin fecha) descubrió que según el evento deportivo, los deportistas que rindieron a un alto nivel en los Juegos Olímpicos, mostraban unas características antropométricas definidas y diferenciadas. Según Strzala y Tyka (2009) a niveles de rendimiento medio-alto, tanto 
los índices biométricos (altura y envergadura), como de estructura corporal (masa magra corporal), en general, son decisivos en la velocidad natatoria.

Una revisión realizada por Faulkner (1966), citado por Siders, Lukaski, y Bolonchuk (1993), indicó que los nadadores velocistas tienden hacia un somatotipo mesomórfico, así como a niveles más bajos de grasa corporal que los nadadores que se dedican a hacer pruebas más largas, los cuales muestran un mayor componente endomorfo (Carter and Marfell-Jones, 1994; citados por Stager y Babington, 1995). Además, muchos estudios coinciden al señalar que estos nadadores tienen una mayor estatura, masa coporal y masa muscular que los nadadores de resistencia (Strzala and Tyka, 2009).

La mayoría de la literatura científica vinculada al estudio de las variables antropométricas y su relación con el rendimiento en la velocidad en natación, se centra en la población de jóvenes nadadores, con el objetivo, por un lado, de encontrar aquellas variables que puedan predecir el rendimiento natatorio a cada edad (Rama, Santos, Gomes, y Alves, 2006), dado que en edades tempranas, los atributos relacionados con el éxito deportivo varían en función de la edad del deportista (Stager y Babington, 1995). Por otro lado, se pretende establecer un criterio en el que basarse para el proceso de identificación de talentos y de desarrollo en un deporte concreto (Blanksby, Bloomfield, Ponchard, y Ackland, 1986). En este sentido, se han encontrado determinados parámetros antropométricos, como la masa corporal, masa muscular, altura y envergadura; que correlacionan positivamente con el rendimiento en las pruebas de velocidad de natación, por lo que deben ser tenidas en cuenta (Geladas et al., 2005; Grimston and Hay, 1986; Jürim.e et al., 2007, Silva et al., 2007; citados por Lätt et al., 2010). Esto puede deberse a la relación que guardan dichas variables con la longitud de brazada (Strzala and Tyka, 2009), la cual juega un papel decisivo en una prueba de 50 metros (Girold et al., 2012).

Son varios los estudios que han destacado la importancia de la altura y de la envergadura de brazos para el rendimiento en las pruebas de velocidad. Borms (1986), citado por Camarero, Tella, Moreno, y Fuster (sin fecha), refuerza el concepto sobre la importancia de la altura en los nadadores, indicando, en su revisión bibliográfica, el interés de los parámetros antropométricos como son la longitud de los miembros superiores e inferiores y las longitudes de las manos y de los pies. Por otro lado, Cardoso y Alves (1995), citados por Camarero et al. (sin fecha), llegaron a la conclusión de que la técnica de crol muestra una relación positiva con la altura, envergadura y longitud de la mano. En esta misma línea, Nasirzade, Ehsanbakhsh, Argavani, Sobhkhiz, y Aliakbari (2014), evaluaron el rendimiento del 50 libre en 23 nadadores de entre 13 y 14 años, con una experiencia competitiva de entre 4 y 6 años, llegando a la conclusión de que el tiempo empleado en realizar la prueba está relacionado de forma significativa con la altura y la envergadura. Por otro lado Zampagni, Casino, Benelli, Visani, Marcacci, y De Vito (2008), en un intento de determinar cuáles son las variables que pueden predecir el rendimiento en las pruebas de estilo libre en nadadores master de entre 40 y 80 años, indicaron que la altura fue una de los mejores predictores en las pruebas de 50 y 100 libres.

Por otro lado, se ha demostrado que la longitud de las extremidades superiores también juega un papel relevante en el rendimiento en las pruebas de velocidad. Geladas, Nassis, y Pavlicevic (2005) llegaron a la conclusión de que la longitud de las extremidades superiores, puede ser usada para predecir el rendimiento en los 100 metros libre en chicos de entre 12 y 14 años. Igualmente, Lätt et al. (2010), sugieren que, debido a la correlación entre el rendimiento en los 100 metros libre y la envergadura, la longitud de las extremidades superiores y la anchura de hombros, 
pueden estar relacionadas con factores biomecánicos relevantes para la propulsión. La explicación a este hecho puede deberse a que cuanto más larga sea la extremidad, mayor será la longitud de los fascículos musculares. Dicha longitud, correlaciona positivamente con la velocidad de acortamiento del músculo (Lieber y Friden, 2000), además, podría dar lugar a una mayor generación de potencia muscular, lo que a su vez mejoraría el rendimiento en las pruebas de sprint (Kumagai et al., 2000; citados por Nasirzade et al., 2014).

Autores como Klika y Thorland (1994), identificaron otras variables relacionadas con la composición corporal que se asociaron a un mayor rendimiento en la prueba de 100 yardas a crol (91,4 metros). Una de ellas fue la muscularidad (entendida como el nivel de masa muscular en relación a la estatura), la cual correlacionó positivamente con la velocidad de nado en dicha prueba en un grupo de nadadores de entre 17 y 22 años, así como con otras variables como el volumen y la masa corporal, masa muscular, potencia en los miembros inferiores y fuerza en la brazada. Por otro lado, encontraron que la densidad corporal (masa corporal en relación al volumen corporal), además de correlacionar negativamente con el nivel de grasa corporal, correlacionó de forma significativa con la fuerza de brazada, dejando ver que niveles más altos de densidad corporal contribuyen a nadar más rápido por este hecho.

\section{LA FUERZA Y SUS DISTINTAS MANIFESTACIONES}

Desde la perspectiva de la actividad física y del deporte, podemos definir la fuerza como la capacidad de un músculo o grupo muscular para vencer o soportar una resistencia bajo unas condiciones específicas (Siff y Verhoshansky, 2000; García Manso et al, 1996; citados por Cancela y Ramírez, sin fecha), siendo considerada como una de los factores más importantes que determinan el éxito deportivo (Özçaldiran, y Durmaz, 2008).

En natación, mediante la generación de tensión muscular, el deportista transfiere una determinada cantidad de energía cinética al agua, acelerando cierta cantidad de masa de agua y generando fuerza propulsiva (Caputo et al., 2006), la cual representa uno de los factores claves que determinan el rendimiento en el estilo crol (Toussaint, Van der Berg y Beek, 2002). Al contrario de lo que sucede en los deportes en tierra, donde el $100 \%$ de la fuerza que aplica el deportista se traduce en fuerza propulsiva, en natación, dada la inestabilidad del medio en el que se desarrolla, tan solo un porcentaje de la fuerza total aplicada por el nadador servirá para que éste se propulse (Salo y Riewald, 2008). Por esta razón, el desarrollo de la fuerza específica para la mejora del rendimiento presenta una mayor dificultad en este deporte (Salo y Riewald, 2008).

Junto con la velocidad, la fuerza, entendida como la capacidad del músculo para generar la máxima tensión a una velocidad dada (Knuttgen, 1987; citado por Pelot y Darmiento, 2012), pasa a ser un factor determinante en las pruebas cortas de natación (Miyashita y Kanehisa, 1983; Toussaint y Vervoorn, 1990; Tanaka et al., 1993; Trappe y Pearson, 1994; Girold et al., 2007; citado por Garrido et al., 2010), así como uno de los principales elementos a trabajar en el programa de entrenamiento de un velocista (Girold, Calmels, Maurin, Milhau, y Chatard, 2006). De hecho, Watanabe y Takai (2005), citados por Garrido et al. (2010), concluyeron que la fuerza muscular es un importante factor explicativo del rendimiento en pruebas de 50 metros en ambos géneros en nadadores de más de 15 años. 
Por otra parte, parece ser que el desarrollo de la fuerza de los miembros superiores es el factor que más influye en el rendimiento natatorio, dado que las extremidades superiores contribuyen en mayor medida a generar fuerza propulsiva que las inferiores (Girold et al., 2012). De hecho, son varios los estudios que han indicado una correlación significativa entre la fuerza muscular del tren superior y la velocidad de nado en distancias cortas (Toussaint y Vervoorn, 1990; Hawley y Williams, 1991; citados por Garrido et al., 2010), así como con el rendimiento en pruebas de 25 y 50 metros y la fuerza rotacional generada durante el nado (Hawley et al., 1992 y Smith et al., 2002; citados por Garrido et al., 2010). Estas mejoras en la velocidad de nado, pueden ser debidas a la influencia que ejerce la fuerza muscular sobre la longitud de brazada. Parece ser que las ganancias de fuerzas obtenidas a través del entrenamiento, se traducen en un aumento de la longitud de brazada (Toussaint and Vervoorn 1990; citados por Aspenes, Kjendlie, Hoff, y Helgerud, 2009). Este parámetro representa un factor clave en el rendimiento de un 50 libre, a pesar de que la longitud de brazada en esta prueba sea menor en comparación con las pruebas más largas (Girold et al., 2012).

\section{Manifestaciones de la fuerza en natación}

En función de la tensión generada por el músculo, el tiempo que tarde en aplicarse la fuerza, el tipo de contracción producida, además de otros factores; se producirá una determinada manifestación de la fuerza (González-Badillo y Gorostiaga, 1995). En natación, las diferentes manifestaciones activas de la fuerza van a condicionar el rendimiento del deportista en determinados momentos de la prueba (Cancela y Ramírez, sin fecha), aunque suele prestarse especial atención al desarrollo de la fuerza (dinámica) máxima y a la fuerza explosiva, dado que ambas determinan en gran medida la magnitud de la fuerza de tracción que el nadador desarrolla al nadar, además del salto que realizará en la salida (Cancela y Ramírez, sin fecha). Dichos aspectos van a condicionar en buena parte la velocidad de salida y de nado en esta prueba, contribuyendo de forma significativa a la mejora del rendimiento en la misma.

\section{Fuerza máxima}

La fuerza máxima hace referencia a la mayor tensión que puede generar el músculo de forma voluntaria ante una resistencia que puede ser desplazada una sola vez (fuerza dinámica máxima) o cuando ésta no puede ser desplazada (fuerza isométrica máxima) (González-Badillo y Gorostiaga, 1995). En natación, nos centraremos en las contracciones de tipo dinámico, ya que son las que se dan a medida que el nadador avanza por el agua (Garrido et al., 2010).

La mejora de la fuerza máxima es un elemento esencial para la mejora del rendimiento en natación (Aspenes et al., 2009), ya que está relacionada con el incremento de la longitud de ciclo, y por ende, de la velocidad (Trinidad y Lorenzo, 2012). Por otro lado, las ganancias en la fuerza dinámica máxima del deportista van a jugar un papel muy relevante a la hora de retrasar la aparición de la fatiga, dado que a un mismo nivel de contracción submáxima (en términos absolutos), menor será la intensidad relativa (porcentaje de fuerza generada en relación a la máxima capacidad para ejercerla) (Blanksby, Bloomfield, Ponchard y Ackland, 1986; Newton, Jones, Kraemer y Wardle, 2002). Esto quiere decir que el nadador con una mayor fuerza dinámica máxima, empleará menos esfuerzo al aplicar una determinada cantidad de fuerza, fatigándose menos, y por ende, pudiendo mantener con más facilidad los valores óptimos de fuerza y potencia durante más tiempo. Este factor, por tanto, será crucial para mantener la velocidad de nado durante la fase de llegada, es decir, durante los 10 últimos metros de la prueba. 


\section{Fuerza-explosiva}

El concepto de fuerza-explosiva o potencia hace referencia a la capacidad del sujeto de generar fuerza en un tiempo dado, haciendo referencia a la relación entre la fuerza aplicada y el tiempo en el que se aplica dicha fuerza (González-Badillo y Gorostiaga, 1995).

Pelot y Darmiento (2012) indican que, desde la perspectiva del rendimiento en natación, interesa considerar la potencia como la cantidad de trabajo realizado (producto de la fuerza aplicada por el espacio recorrido) en un tiempo concreto (Potencia $=$ trabajo/tiempo). A partir de esta ecuación, podemos deducir que el tiempo empleado en recorrer una determinada distancia, es el cociente del trabajo realizado entre la potencia generada (Tiempo $=$ trabajo/potencia). Por tanto, la única manera de reducir el tiempo empleado en recorrer la distancia de la prueba, es mediante el aumento de la cantidad de potencia generada por el deportista durante dicha prueba.

Por ello, el objetivo de todo nadador, es llegar a ser lo más potente posible, de manera que pueda generar un nivel suficientemente alto de fuerza durante todo el tiempo que dure la prueba para poder terminarla antes que sus competidores (Heusner, 1980; citado por Pelot y Darmiento, 2012). Siguiendo esta línea, si tenemos en cuenta que la fase activa de la brazada (en la cual se produce la fuerza propulsiva) en un sprint realizado a crol dura unos 400 milisegundos (Kolmogorov, y Lyapin, 1999; citados por Dopsaj, Matkovic, y Zdravkovic, 2000), cuanta mayor sea la fuerza que el nadador pueda producir en este tiempo, más rápido se desplazará por el agua (Dopsaj, Matkovic, y Zdravkovic, 2000).

Son muchos los autores que afirman la importante repercusión que tiene la generación de potencia en la velocidad de nado y, por ende, en el rendimiento en la natación en general, y en un 50 libre en particular. Dominguez-Castells, Izquierdo, y Arellano (2013), señalan que la potencia generada por el nadador es un predictor fiable de la velocidad de nado al estilo crol. Además, apuntan que la máxima potencia de nado generada guarda una relación lineal con la máxima velocidad de nado, con independencia de la fatiga o del nivel técnico (Seifert, Toussaint, Alberty, Schnitzler y Chollet, 2010 y Toussaint, Carol, Kranenborg y Truijens; citados por DominguezCastells, Izquierdo y Arellano, 2013). De hecho, los nadadores de nivel mundial producen mayores picos de potencia que los de menor nivel (Barbosa, Morais, Marques, Costa y Marinho, 2014). Por otro lado, Toussaint et al. (2006) citados por Stirn, Jarm, Kapus, y Strojnik (2011), indican que la falta de capacidad para mantener una longitud de brazada y una velocidad de nado constante a lo largo de la prueba, se debe a la incapacidad del deportista para generar los niveles de potencia necesarios que le permitan superar la resistencia del agua.

Tampoco podemos olvidar el importante papel que juega la generación de potencia, especialmente de los miembros inferiores, en el rendimiento de la salida, que como se dijo anteriormente, es un factor clave en el rendimiento de esta prueba (West et al., 2011). Para poder realizar un buen salto, el nadador debe generar una gran potencia a través de la triple extensión de tobillos, rodillas y caderas (Monu, 2013), para poder alcanzar la velocidad de despegue más alta posible, y así, poder conseguir un mayor desplazamiento horizontal de su cuerpo antes de entrar en el agua (Breed y Young, 2003). Según Lee, Huang, y Lee (2013), citados por Bishop, Cree, Read, Chavda, Edwards, y Turner (2013), identificaron que el tiempo que los nadadores permanecían en el bloque de salida fue de 0,79 segundos. Dado que sabemos que este es el tiempo aproximado que el nadador dispone para aplicar la máxima fuerza, podemos tomar dicho valor como referencia a la hora de planificar el entrenamiento de la fuerza-explosiva para mejorar el salto en la salida. 


\section{Potencia y fuerza generada durante un sprint al estilo crol}

Existen varios estudios que, mediante la realización de ciertos tests, han medido la fuerza y la potencia generada por los nadadores durante esfuerzos que se asemejan a aquellos que se llevan a cabo durante un 50 libre. Dominguez-Castells, Izquierdo, y Arellano (2013) midieron la potencia de nado de 18 nadadores de entre 18 y 26 años, en el que tenían que nadar 12,5 metros a un esfuerzo máximo arrastrando varias cargas mediante un sistema de poleas. Se midió la potencia de la brazada con varias cargas, mediante la multiplicación de la fuerza generada y la velocidad alcanzada por el nadador. La máxima potencia de nado de los brazos en términos absolutos y relativos a la masa corporal fue de $66,49(19,09 \mathrm{~W})$ y de $0,86(0,21 \mathrm{~W} / \mathrm{kg})$ respectivamente. La carga externa con la que se alcanzó la máxima potencia de nado fue de $3,95(0,79 \mathrm{~kg})$ y de 47,07 $(9,45 \%)$ con respecto a la máxima carga individual. También descubrieron que existían diferencias en cuanto a la potencia generada durante las distintas fases de la brazada. Durante la fase de empuje (parte final de la brazada) la potencia fue de $71,21(21,06 \mathrm{~W})$, mientras que la registrada durante la fase de tirón (fase media de la brazada), fue de 60,32 (33,16 W). Johnson, Sharp y Hedrick, (1993), midieron el pico máximo de potencia de 29 nadadores de entre 14 y 22 años. Para ello, los sujetos nadaron a la máxima velocidad posible con un cable atado a la cintura, el cual iba a conectado a un dispositivo que registraba la potencia generada por el nadador. La media en el pico de potencia generado por estos nadadores fue de $85 \mathrm{~W}$. Esta variable correlacionó positivamente con la velocidad de nado en un test de 25 yardas (22,85 metros) realizado al máximo esfuerzo. Por otra parte, utilizando métodos de medición de la potencia de nado en seco, Bradshaw y Hoyle (1993), midieron la potencia media generada en siete nadadores universitarios con al menos tres años de experiencia en la natación competitiva. Para ello, utilizaron un banco de natación que simulaba el patrón de movimiento realizado durante el nado crol. Los valores medios registrados fueron de 93,13 W (en términos absolutos), con un promedio de 20,4 brazadas.

El único estudio que hemos encontrado en el que se cuantificó la fuerza producida durante un sprint a estilo crol es el de Peyrebrune, Toubekis, Lakomy, Nevill (2012). Para ello, utilizaron el método en el cual el sujeto debe nadar con un cable atado a su cintura. La fuerza media producida por los participantes en un sprint de 30 segundos fue de $139(13 \mathrm{~N})$, mientras que el pico máximo de fuerza se alcanzó a los 2 segundos del inicio del test (186 (16 N)). Los niveles de aplicación de fuerza fueron disminuyendo progresivamente hasta llegar a $113(13 \mathrm{~N})$ durante la segunda parte de la prueba.

\section{PERFIL DE RESISTENCIA AERÓBICO}

Si bien es cierto que cuanto más larga sea la distancia de la prueba, mayor importancia cobrará la capacidad de resistencia aeróbica (Chatard y Stewart, 2011; citados por Holfelder, Brown, Bubeck, 2013), el papel que juega el metabolismo aeróbico a la hora de suministrar energía en una prueba de tan corta duración como es el 50 libre, no debería ser subestimado (Ring, Mader, Wirtz, Wilke, 1996).

En el trabajo de Peyrebrune, Toubekis, Lakomy, y Nevill (2012), en el que se midió la contribución de los sistemas energéticos en el suministro de energía durante un sprint máximo de 30 segundos a partir del déficit de oxígeno acumulado tras la realización del esfuerzo, se descubrió que el metabolismo aeróbico contribuyó considerablemente (en torno a un 33\%) a la producción de trabajo durante el tiempo que duraba el test. Según estos mismos autores, los resultados obtenidos coinciden con otros trabajos previos, aunque señalan que la contribución de los distintos sistemas energéticos durante este las pruebas de velocidad, dependerá del tiempo 
que se tarde en completar la misma. Valores más bajos fueron observados por Ring, Mader, Wirtz, y Wilke (1996), quienes llegaron a la conclusión de que el sistema aeróbico aportaba entre el 17,8 y el $29,1 \%$ de la energía necesaria para completar una carrera de 50 metros crol. En cualquier caso, el oxígeno empleado durante esta prueba procede principalmente de las reservas del organismo, dado que el número de respiraciones durante la misma es mínimo (Hermansen, 1981; Saltin y Essem, 1971; citados por Ring, Mader, Wirtz, Wilke, 1996).

Por otro lado, la contribución de los diferentes sistemas energéticos variará a lo largo de la prueba. Bogdanis et al. (1998), citados por Peyrebrune, Toubekis, Lakomy, y Nevill (2012) señalan que será en la última parte de la prueba cuando el metabolismo aeróbico comience a adquirir más peso en el aporte de energía, lo que se debe a un gran descenso de los niveles de fosfocratina y a la incapacidad de la glucolisis anaeróbica para producir altas cantidades de ATP (Hirvonen, Rehunen, Rusko y Harkonen, 1987; citados por Ring, Mader, Wirtz, y Wilke, 1996). De hecho, durante los primeros 15, la contribución del sistema aeróbico será de entre el 3 y el $5 \%$ según los resultados obtenidos por Ring, Mader, Wirtz, y Wilke (1996).

\section{Consumo de oxígeno y consumo máximo de oxígeno durante la prueba}

En la natación, el consumo máximo de oxígeno, se considera un factor importante que influye en el rendimiento (Fernandes et al. 2008). Pero en el 50 libre, podemos decir que no juega un papel tan determinante en el rendimiento, ya que al ser una prueba de muy corta duración, el nadador no tiene tiempo de alcanzar su máximo consumo de oxígeno, el cual se estima que se alcanza, según los resultados obtenidos del estudio de Fernandes et al. (2008), sobre los 4 minutos. Este hecho también se debe a que se trata de un esfuerzo que se realiza prácticamente en condiciones de hipoxia, y se ha demostrado que un esfuerzo realizado en dichas condiciones, conlleva a consumos de oxígeno más bajos (Engelen, 1996; Hughson, 1995; citados por Ogita, 2006).

Peyrebrune, Toubekis, Lakomy, y Nevill (2012), registraron un consumo de oxígeno de $33,2(7,1)$ mililitros por kilo de peso y por minuto, en un sprint de 30 segundos realizado con una cuerda atada a la cintura. Estos valores se correspondieron con un consumo de oxígeno del 59 (10\%) con respecto al consumo máximo de oxígeno del deportista. Por otro lado, Ring et al. (1996), indicaron que los nadadores especializados en distancias cortas, alcanzaron valores de consumo de oxígeno que se correspondían con el $74 \%$ de su consumo máximo de oxígeno tras realizar 50 metros a crol al máximo esfuerzo.

\section{PERFIL DE RESISTENCIA ANAERÓBICA}

Aunque sabemos que la contribución de todos los sistemas energéticos va a determinar la capacidad de rendimiento en natación (Toussaint y Hollander 1994; citados por Rodríguez y Mader, 2003), el papel de los sistemas aneróbicos (láctico y aláctico) en las pruebas de sprint (50 y 100 metros), es especialmente decisivo (Sharp, Troup, Costill, 1982). Además, la capacidad del sujeto de producir ATP por unidad de tiempo a través de estos sistemas, lo que se conoce como potencia anaeróbica, será un factor crucial para el rendimiento en esta prueba (Issurin, Kaufman y Tenenbaum, 2001; Vandewalle, Pérès, Sourabié, Stouvenel y Monod, 1989).

Los datos recogidos por Peyrebrune, Toubekis, Lakomy, y Nevill (2012), indicaron una contribución del $67(8 \%)$ por parte del metabolismo aneróbico durante un sprint de 30 segundos. Los resultados de Zamparo, Capelli, Cautero, y Di Nino (2000), 
mostraron que los sistemas anaeróbicos eran los responsables de suministrar el 73,2 $(6,1 \%)$ de la energía total en un sprint máximo al estilo crol de 32 segundos. Por otro lado, Capelli, Pendergast, y Termin (1998), indicaron que durante un sprint a crol de 25 segundos, el metabolismo anaeróbico era responsable de proporcionar ATP en un $84,7 \%$, siendo el sistema anaeróbico láctico el que más energía produce $(58,9 \%)$, seguido del sistema anaeróbico aláctico $(25,8 \%)$.

En base a estos resultados, podemos decir que el sistema glucolítico es el que predomina en un 50 libres, empezando a activarse de forma clara, según Ring et al. (1996), a partir de los 6 segundos del comienzo de la prueba aproximadamente. La contribución de dicho sistema es fundamental para la refosforilación de la fosfocreatina, lo que se traduce en una mayor disponibilidad de ATP para el trabajo muscular (Ring et al., 1996).

Como consecuencia de las altas demandas que se imponen sobre el sistema glucolítico en esta prueba, se producen elevadas concentraciones de lactato (Strzala and Tyka, 2009), que es un subproducto generado por este tipo de sistema, y que es el responsable de que aparezca la fatiga muscular en los últimos metros de la prueba. Las altas concentraciones de lactato disminuirán la capacidad de los músculos para producir fuerza, mermando la capacidad del nadador de mantener una óptima longitud de brazada (Stirn, Jarm, Kapus y Strojnick, 2011), y alterando su técnica y su mecánica de brazada, desembocando en una reducción de la velocidad de nado (Castro y Mora, 2008; citados por Trinidad y Lorenzo, 2012; Stirn et al., 2011).

Capelli et al. (1998), obtuvieron valores de lactato de $9,9 \mathrm{mmol} / \mathrm{L}$ en 8 nadadores que realizaron un sprint a crol de 45,7 metros a una velocidad media de $1,97 \mathrm{~m} / \mathrm{s}$. Por otro lado, Ring et al. (1996), observaron que el lactato producido por nadadores especializados en pruebas de velocidad durante un sprint de 50 metros crol, de 28 segundos de duración, fue de $7 \mathrm{mmol} / \mathrm{l}$., alcanzándose la máxima producción de lactato por unidad de tiempo a los 12 segundos $(0,9 \mathrm{mmol} / \mathrm{s})$ del comienzo del esfuerzo. Las diferencias observadas entre ambos estudios en cuanto a las concentraciones de lactato en sangre post-ejercicio, pueden deberse, entre otros cosas, a los distintos tiempos en los que se tomaron las muestras.

\section{VALORACIÓN DEL RENDIMIENTO}

Para conseguir el máximo rendimiento en las competiciones, un diagnóstico regular del mismo se convierte en un requisito imprescindible (Olbrecht, 2011; citado por Holfelder, Brown, y Bubeck, 2013). La importancia de una evaluación periódica del rendimiento radica, en primer lugar, en la posibilidad de determinar el nivel de preparación del deportista en relación a las cargas de entrenamiento a las que se ve sometido, lo que nos permitirá controlar y regular el proceso de entrenamiento (Cechovská, 1994; citado por Ruzbarsky y Turek, 2006). En segundo lugar, la evaluación nos permitirá determinar de forma objetiva el posible resultado del nadador en la competición (Anderson, Hopkins, Roberts, y Pyne , 2008: citados por Fernandes, Sousa, Machado y Vilas-Boas, 2011).

A continuación, se presentan diferentes tests que se utilizan en el ámbito del entrenamiento de la natación, los cuales se emplean como herramienta para evaluar los distintos componentes del rendimiento en este deporte. 


\section{Valoración de la fuerza-explosiva}

Desafortunadamente, son escasos los métodos de evaluación específicos de la potencia muscular desarrollados hasta el momento en el campo de la natación (Swaine, 2000; citados por Garrido et al., 2010). Las mediciones de la fuerza-explosiva que se utilizan en el ámbito de la natación, pueden llevarse a cabo en seco (fuera de la piscina), o bien en el agua, donde el nadador deberá nadar a un máximo esfuerzo con una cuerda atada a su cintura (Smith, Norris y Hoggs, 2002)

Uno de los test que suelen emplearse en seco para medir la potencia de los miembros inferiores, por su relación con el rendimiento en la salida (Pearson et al., 1998; Zatsiorsky, Bulgakova y Chaplinsky, 1979; citados por Rebutini et al., 2014), es el test de salto vertical, más concretamente el "squat jump", considerándose como el instrumento más apropiado para medir la potencia en nadadores especializados en pruebas de velocidad (Bishop et al., 2013).

Para evaluar la potencia generada durante el nado, lo más recomendable es emplear tests que se realicen en condiciones específicas, es decir, en los que el nadador reproduzca los patrones de movimiento propios de la modalidad deportiva, y lo que es más importante, que se lleven a cabo en el medio acuático (Muller, Benko, Raschner, y Schwameder, 2000; citados por Smolka y Ochmann, 2013). Por un lado, nos encontramos con aquellos tests que miden la potencia de nado en la propia piscina, en los que el deportista deberá nadar al máximo esfuerzo con una cuerda atada a su cintura, registrándose la potencia a través de un dispositivo. Por otro lado, se encuentran aquellos que se realizan en tierra, empleando un banco que intenta simular el patrón de movimiento de la brazada (Johnson, Sharp y Hedrick, 1993).

Estos últimos, comprenden aquellas pruebas específicas realizadas en ergómetros diseñados para imitar la acción de los brazos durante el nado, en los que se implica a la gran mayoría de los grupos musculares que se emplean durante un sprint en el agua (Costill, Maglischo, y Richardson, 1992; citados por Smith, Norris y Hoggs, 2002). Aun así, se ha de decir que dichos bancos no replican de manera exacta la técnica de brazada que el nadador utiliza cuando nada (Bradshaw y Hoyle, 1993), por lo que los tests que se realicen en él no son tan recomendables como aquellos que se llevan a cabo en el agua.

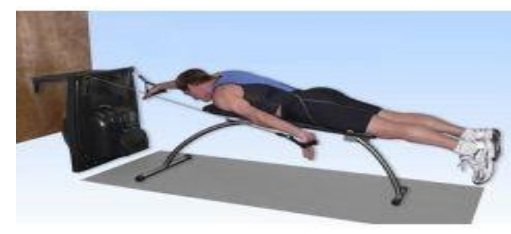

Figura 1: Ejemplo de banco de natación

Sharp et al. (1982), llevaron a cabo un estudio en el que demostraron que existía una correlación positiva entre la potencia desarrollada durante una tracción máxima realizada sobre el banco y la velocidad de nado en un sprint de 22,75 metros. El test que utilizaron estos autores para medir la potencia de nado se llevó a cabo en un banco de natación biocinético, al cual se le podía regular la velocidad, de tal manera que se pudiera generar una cantidad constante de aceleración en proporción directa a la fuerza aplicada por el nadador. El nadador, se coloca en decúbito-prono, con una correa alrededor de sus caderas que le sujeta. Las manos las colocan en unas palas que trae el aparato, quedando con los brazos totalmente extendidos por encima de su cabeza. Después de que el sujeto se familiarice con el ergómetro, deberá realizar una 
tracción máxima para cada una de las velocidades programadas, quedando registrada la potencia generada.

Sin embargo, dado que los mejores nadadores no necesariamente tienen que producir los mejores resultados en estos tipos de ergómetros, es más recomendable utilizar tanto la máxima velocidad de nado como la fuerza producida en el nado para evaluar de forma conjunta el rendimiento técnico y de fuerza-explosiva en el agua (Keskinen, 1994; citado por Smith, Norris y Hoggs, 2002). Para ello, se utilizan los denominados "tethered swimming tests", que son tests en los que el nadador, con una cuerda atada a su cintura, deberá realizar un esfuerzo máximo, permaneciendo en el mismo sitio sin avanzar, es decir, a velocidad cero.

Un ejemplo en el que se mide la potencia de nado en el agua utilizando esta metodología, lo encontramos en el test que proponen Domínguez-Castells, Izquierdo y Arellano (2013). El objetivo de dicho test será examinar la potencia intra-ciclo en las fases propulsivas y determinar tanto la potencia máxima de nado como la velocidad a la que se alcanza dicha potencia. Para ello, el sujeto nada a un esfuerzo máximo durante 12,5 metros, propulsándose sólo con sus brazos y con una cuerda atada a su cintura. Mediante un encóder lineal y una célula de carga, se registra la velocidad y la fuerza en cada ciclo de brazada. En base a esos datos, se obtiene una curva de la potencia generada en cada ciclo durante la fase propulsiva de la brazada (fase subacuática de la brazada), registrándose la potencia media, la potencia máxima, así como la velocidad de nado a la que se alcanza dicho valor.

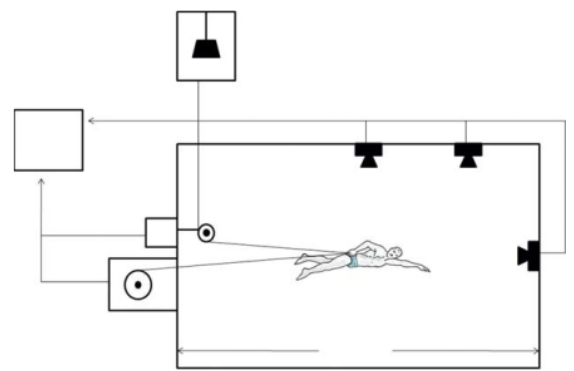

Figura 2: Representación gráfica del test empleado por Domínguez-Castells, Izquierdo, y Arellano (2013) para evaluar la potencia de la brazada en cada ciclo.

Estos autores, observaron una correlación positiva entre la máxima potencia de nado y la velocidad máxima de nado conseguida en un sprint de 25 metros. En vista a los resultados obtenidos, llegaron a la conclusión de que este test, puede ser una herramienta para el entrenamiento dada la facilidad para poder implementarlo, proporcionando a los nadadores y entrenadores un feedback inmediato.

\section{Valoración de la fuerza máxima}

La capacidad de contracción máxima del nadador fuera del agua, también puede ser evaluada por los entrenadores con el fin de monitorizar la progresión del deportista y de predecir alguno de los componentes del rendimiento. Aun así, son pocos son los estudios que han investigado la relación que puede haber entre la fuerza máxima fuera del agua y el rendimiento natatorio.

West et al. (2011) encontraron una relación significativa entre el test de 1RM en sentadilla y el rendimiento en la salida (primeros 15 metros) en 11 nadadores velocistas de primer nivel. Para ello, determinaron la carga con la que los nadadores podían hacer un máximo de tres repeticiones (3RM), y en base a ese resultado, estimaron la RM a partir de las tablas proporcionadas por Baechle y Earle (2000). 
Para medir la fuerza máxima del tren superior, los test de campo que más se utilizan a día de hoy en el entrenamiento de la natación son el test de 1RM en el press de banca y el de 1RM en dominada (Bishop et al., 2013). Dichos test son elegidos, en primer lugar, porque activan en conjunto prácticamente los mismos grupos musculares que cuando se nada (Cronin et al., 2007; citados por Garrido et al., 2010), y en segundo lugar, porque al ser ejercicios multiarticulares, podría resultar beneficioso a la hora de estudiar su relación con un movimiento dinámico como el del nado (Garrido et al., 2010). Además, según el estudio de Garrido et al (2010), el test de fuerza realizado en el press banca guarda una correlación moderada con el rendimiento en las pruebas de 25 y 50 libres. Sin embargo, Johnson, Sharp, y Hedrick (1993), llegaron a la conclusión de que el test de la RM en press de banca no era un buen predictor del rendimiento en el sprint a crol.

Girold et al. (2006), en su estudio, descubrieron que la fuerza isométrica de los flexores y extensores del codo representa un buen predictor para el rendimiento en la prueba de 100 libre. Para evaluar dicha variable emplearon un dinamómetro isocinético. Antes de que el nadador realizase el test, se programó el dinamómetro a diferentes velocidades angulares (60,180 y $0 \%$ s), ya que éstas parecen ser las más representativas de la velocidad del movimiento del nadador.

\section{Valoración de la resistencia aeróbica}

En la natación, la resistencia aeróbica representa uno de los indicadores más importantes del nivel de forma física del deportista (Jorgić, Puletić, Okičić, y Meškovska, 2011), y su monitorización periódica, junto con la de la resistencia anaeróbica, ofrece al entrenador la oportunidad de controlar y adaptar el entrenamiento de forma apropiada (Strzala and Tyka, 2009).

Uno de los parámetros funcionales más básicos para su medición es el consumo máximo de oxígeno (VO2 máx.) (Ruzbarsky y Turek, 2006), el cual se ha mostrado especialmente relevante para las pruebas de entre 400 y 1500 metros. Aunque el VO2 máx. no es uno de los aspectos esenciales del rendimiento para una prueba tan corta como el 50 libre, es importante determinarlo, ya que según los valores del mismo, se podrá monitorizar de forma óptima la intensidad de la carga de entrenamiento a través de la frecuencia cardíaca (FC) y la percepción subjetiva del esfuerzo (Jorgić, Puletić, Okičić, y Meškovska, 2011)

En natación, la determinación del VO2 máx. suele hacerse mediante tests en los que el deportista nada una serie de intervalos repetidos a una velocidad cada vez mayor, hasta llegar al punto en el que el VO2 se estabiliza (Maglischo, 2003; citado por Jorgić, Puletić, Okičić, y Meškovska, 2011).

Las medidas del VO2 pueden tomarse de forma directa, esto es, a través de un analizador de gases en los que se registra el oxígeno consumido y el dióxido de carbono expulsado (Holfelder, Brown, Bubeck, 2013); o bien de forma indirecta, basándose en la relación linear existente entre la FC y el oxígeno consumido a intensidades submáximas (Jorgić, Puletić, Okičić, y Meškovska, 2011). Aprovechando esta relación, Smith, Norris, y Hoggs (2002) señalan que, debido a la dificultad de medir el VO2 en la gran mayoría de las situaciones de entrenamiento por falta de equipamiento, se llevan a cabo una serie de "tests de campo" basados en el registro de la FC a diferentes velocidades de nado. Los resultados obtenidos de estos tests nos indican aspectos tales como la economía de nado (capacidad de nadar a una misma intensidad con un menor gasto de energía, o a una mayor intensidad con el mismo gasto de energía), los estados transitorios o más duraderos de la fatiga, y el nivel de preparación del nadador. 
Salo y Reiwald (2008), proponen un test cuyo objetivo es evaluar el estado de forma del nadador. Consiste en nadar una serie de 8 repeticiones de 100 metros, con una recuperación de 4 minutos entre repeticiones. Empezando la primera repetición a una intensidad del $70 \%$, se aumentará la intensidad de nado en un $10 \%$ en cada repetición hasta llegar a la repetición cuarta (la cual se realizará a la máxima intensidad), y a partir de la quinta - que también se realizará a la máxima intensidad la intensidad se irá reduciendo en un $10 \%$ en cada repetición hasta llegar a la octava.

Después de cada repetición, el nadador deberá tomarse el pulso en la arteria carótida a los 10,30 y 60 segundos de haber acabado la repetición. Se deberá registrar el número de latidos en 10 segundos en cada una de las tres veces que el nadador se tome el pulso, para posteriormente sumarse.

Posteriormente, se elaborará una gráfica en la que aparecerá en el eje de ordenadas la frecuencia cardíaca total (suma de las tres mediciones de la FC tomadas después de cada repetición), y en el eje de abscisas, la velocidad de nado de cada repetición, la cual se obtiene dividiendo la distancia recorrida (100 metros) por el tiempo empleado en completarla.

A medida que mejora el nivel de rendimiento, la gráfica se desplazará hacia la derecha. Esto quiere decir que, para mantener una determinada velocidad de nado, la frecuencia cardíaca será más baja, o que, a una misma frecuencia cardíaca, el nadador es capaz de realizar las repeticiones en menos tiempo. Por tanto, en función del desplazamiento que adquiera la gráfica, podremos determinar si el deportista va adaptándose a las cargas de entrenamiento o no.

Otro indicador que se utiliza en natación para el diagnóstico de la resistencia aeróbica es el umbral anaeróbico (Fernandes et al., 2011), que es el punto a partir del cual los sistemas anaeróbicos empiezan a predominaren la producción de energía para la actividad muscular. Para determinar dicho umbral, es común en el campo del entrenamiento de la natación, registrar los valores de lactato en sangre en cada uno de los tests que se lleven a cabo (Holfelder, Brown, Bubeck, 2013). Madsen y Lohberg (1987), citados por Fernandes et al. (2011), abogan por la utilización de este método para evaluar la capacidad aeróbica, debido a que puede ser realizado de forma rápida, fácil y precisa en la propia piscina, permitiendo obtener los resultados de forma inmediata.

Fernandes et al. (2011), proponen un test para evaluar el umbral anaeróbico del nadador a través del modelo de la curva velocidad-lactato (relación que se establece entre la velocidad de nado y las concentraciones de lactato sanguíneo a cada velocidad de nado). Consiste en realizar 7 repeticiones de 200 metros, con 30 segundos de recuperación entre cada repetición, en los que se va aumentando la velocidad en cada repetición. Se intentará que la velocidad de la última repetición se corresponda con la velocidad media que se consigue en un 400 libre a realizado a un máximo esfuerzo. Las tomas de muestra de las concentraciones de lactato se obtendrán del lóbulo de la oreja del deportista, y se realizan en cada uno de los intervalos de descanso entre repeticiones, al finalizar el ejercicio, 1 minuto después y 3 minutos después de la última repetición.

\section{Valoración de la resistencia anaeróbica}

Una evaluación eficaz de la potencia y de la capacidad anaeróbica constituye un aspecto importante en la evaluación de un nadador (Smith, Norris y Hoggs, 2002), especialmente para aquellos especializados en las pruebas de velocidad, donde la efectividad de los procesos anaeróbicos van a jugar un papel decisivo en el 
rendimiento (Issurin, Kaufman y Tenenbaum, 2001; Vandewalle, Pérès, Sourabié, Stouvenel y Monod, 1989).

Sin embargo, en el campo de la investigación de este deporte, aún no se ha encontrado un método fiable con el que evaluar las habilidades anaeróbicas del deportista (Soares et al., 2006; Smith, Norris y Hoggs, 2002). El procedimiento más frecuente que se ha venido realizando para medir el rendimiento anaeróbico, tanto en natación como en otros deportes, es el Wingate Test (WAnT) (Strzala y Tyka, 2009), el cual consiste en pedalear (con los brazos, para medir la potencia del tren superior, o con las piernas, para medir la potencia del tren inferior) al máximo esfuerzo durante 30 segundos, registrándose la máxima potencia generada, la potencia media y el índice de fatiga.

Hawley y Williams (1991), encontraron una relación significativa entre la potencia anaeróbica del tren superior medida a través de este test, y el rendimiento en las pruebas de 50 metros. Por ello, indican que este test puede ser una herramienta útil para valorar y monitorizar este parámetro en nadadores competitivos. Por otro lado, Strzala y Tyka (2009), emplearon este test, pero con una duración de 60 segundos en lugar de 30, para medir la resistencia anaeróbica tanto de los brazos como de las piernas (de forma separada). Los resultados mostraron que la potencia máxima obtenida en dicho test correlacionó con la velocidad de nado en las pruebas de 25 y 100 metros.

Sin embargo, otros autores, como Soares et al. (2006), señalan que el uso de este test para evaluar el rendimiento anaeróbico en natación es poco adecuado, dado que no se realiza en las condiciones específicas propias de la modalidad deportiva. En esta misma línea, Guilherme, Guglielmo, y Denadai (2000), no encontraron ningún tipo de correlación entre los resultados obtenidos en el WAnT (realizado con los brazos) y la máximas velocidades de nado en pruebas de 14, 25, 50 y 400 metros realizadas al estilo crol.

En busca de una mayor especificidad, y basándonos en el WAnT, podemos llevar emplear tests en los que el nadador tenga que realizar un esfuerzo máximo durante un tiempo determinado en el ergómetro de natación del que hablábamos anteriormente. Los valores de potencia media obtenidos durante el tiempo que dure el test, pueden indicarnos la potencia o la capacidad anaeróbica del deportista. Un test de estas características fue utilizado en el estudio de Brandshaw y Hoyle (1993), donde los nadadores tuvieron que realizar un esfuerzo máximo en un ergómetro de natación, regulado a una velocidad de $1 \mathrm{~m} / \mathrm{s}$. El tiempo que duraba el test era el mismo que el tiempo empleado por el nadador en recorrer 25 metros en la piscina sólo propulsándose con sus brazos. La potencia media generada en este test correlacionó positivamente con el rendimiento en un sprint de 25 metros en el que el nadador sólo utilizaba sus brazos para propulsarse.

Con el mismo objetivo de dar una solución a la falta de especificidad del WAnT, Smolka y Ochmann (2013), proponen un test específico en el agua, basado en el WanT, para valorar el rendimiento anaeróbico. Para ello, el nadador deberá nadar 100 metros a un esfuerzo máximo, esto es, intentando recorrer la distancia en el menor tiempo posible, registrándose la velocidad instantánea del mismo (en franjas de tiempo de 0,02 segundos), dando lugar a una curva de velocidad, que nos indicará los cambios que se producen en la velocidad de nado a lo largo del test. A partir de dichos cambios, según estos autores, se puede inferir el rendimiento anaeróbico con más precisión que cuando se obtienen únicamente los valores de la velocidad media, ya que se podrán obtener parámetros relacionados con el rendimiento anaeróbico tales 
como la velocidad máxima, el tiempo que tarda en alcanzarse la velocidad máxima, tiempo que puede mantenerse la velocidad máxima, la velocidad más baja o el porcentaje de descenso de la velocidad.

Por último, se encuentran los tests de velocidad más simples, basados en el tiempo que emplea el nadador en recorrer una determinada distancia. El trecho a recorrer dependerá de la cualidad que queramos evaluar (potencia anaeróbica láctica, capacidad anaeróbica láctica, etc.). Un test que propone Brandshaw y Hoyle (1993), es el de realizar 9 repeticiones de 25 metros en el siguiente orden: 3 a nado completo, 3 propulsándose sólo con las piernas, y 3 sólo con los brazos. El nadador deberá comenzar el test desde el agua, impulsándose desde la pared, mientras que la recuperación entre repeticiones deberá ser completa.

Arellano Colomina, R., Brown, P., Cappaert, J., y Nelson, R. C. (1994). Analysis of 50-, 100-, and 200-m Freestyle Swimmers at the 1992 Olympic Games.

Arellano, R., Pardillo, S., y Gavilán, A. (2002). Underwater undulatory swimming: Kinematic characteristics, vortex generation and application during the start, turn and swimming strokes. In Proceedings of the XXth International Symposium on Biomechanics in Sports, Universidad de Granada.

Aspenes, S., Kjendlie, P. L., Hoff, J., y Helgerud, J. (2009). Combined strength and endurance training in competitive swimmers. Journal of sports science \& medicine, 8(3), 357-365.

Barbosa, T. M., Morais, J. E., Marques, M. C., Costa, M. J., y Marinho, D. A. (2014). The power output and sprinting performance of young swimmers. Journal of strength and conditioning research/National Strength \& Conditioning Association. Bishop, D. C., Smith, R. J., Smith, M. F., y Rigby, H. E. (2009). Effect of plyometric training on swimming block start performance in adolescents. The Journal of Strength \& Conditioning Research, 23(7), 2137-2143.

Bishop, C., Cree, J., Read, P., Chavda, S., Edwards, M., y Turner, A. (2013). Strength and Conditioning for Sprint Swimming. Strength \& Conditioning Journal, 35(6), 16.

Blanksby, B.A., Bloomfield, J., Ponchard, M., y Ackland, T.R. (1986). The relationship between anatomical characteristics and swimming performance in state agegroupchampionship competitors. Journal of swimming research, 2(2), 30-36.

Bradshaw, A., y Hoyle, J. (1993). Correlation between sprinting and dryland power. Journal of Swimming Research, 9, 15-18.

Breed, R. V., y Young, W. B. (2003). The effect of a resistance training programme on the grab, track and swing starts in swimming. Journal of sports sciences, 21(3), 213220.

Cancela, J.M. y Ramírez, E. (sin fecha). La formación de jóvenes nadadores. Evolución de la composición corporal y de los niveles de fuerza de desplazamiento en nadadores/as brasileños/as de edad comprendida entre los 13 y 23 años.

Camarero, S., Tella, V., Moreno, J.A., y Fuster, M.A. (sin fecha) Perfil antropométrico en las pruebas de 100 y 200 libres.

Capelli, C., Pendergast, D. R., y Termin, B. (1998). Energetics of swimming at maximal speeds in humans. European journal of applied physiology and occupational physiology, 78(5), 385-393. 
Caputo, F., Oliveira, M., Denadai, B. S., y Greco, C. C. (2006). Intrinsic factors of the locomotion energy cost during swimming. Revista Brasileira de Medicina do Esporte, 12(6), 399-404.

Cejuela Anta, R., Pérez Turpin, J. A., Villa Vicente, J. G., Cortell Tormo, J. M., y Rodríguez Marroyo, J. A. (2007). Análisis de los factores de rendimiento en triatlón distancia sprint. Journal of human sport and exercise, 2(2), 1-25.

Dominguez-Castells, R., Izquierdo, M., y Arellano, R. (2013). An updated protocol to assess arm swimming power in front crawl. International Journal of Sport Medicine, 34, 324-329.

Dopsaj, M., Matković, I., y Zdravković, I. (2000). The relationship between 50mfreestyle results and characteristics of tethered forces in male sprint swimmers: A new approach to tethered swimming test. In Facta Universitatis, Series: Physical Education and Sport, 1(7), 15-22.

Fernandes, R. J., Sousa, M., Machado, L., y Vilas-Boas, J. P. (2011). Step length and individual anaerobic threshold assessment in swimming. International Journal Of Sports Medicine, 32(12), 940-946.

Figueiredo P, Zamparo P, Sousa A, Vilas-Boas J. P, y Fernandes R. J. (2011). An energy balance of the $200 \mathrm{~m}$ front crawl race. European Journal of Applied Physiology, 111, 767-777.

Garrido, N., Marinho, D. A., Barbosa, T. M., Costa, A. M., Silva, A. J., Pérez Turpin, J. A., y Marques, M. C. (2010). Relationships between dry land strength, power variables and short sprint performance in young competitive swimmers. Journal of Human Sport \& Exercise, 5(2), 240-249.

Geladas, N. D., Nassis, G. P., y Pavlicevic, S. (2005). Somatic and physical traits affecting sprint swimming performance in young swimmers. International Journal of Sports Medicine, 26(02), 139-144.

Girold, S., Calmels, P., Maurin, D., Milhau, N., y Chatard, J. C. (2006). Assisted and resisted sprint training in swimming. The Journal of Strength \& Conditioning Research, 20(3), 547-554.

Girold, S., Maurin, D., Dugue, B., Chatard, J. C., y Millet, G. (2007). Effects of dryland vs. resisted-and assisted-sprint exercises on swimming sprint performances. The Journal of Strength \& Conditioning Research, 21(2), 599-605.

Girold, S., Jalab, C., Bernard, O., Carette, P., Kemoun, G., y Dugué, B. (2012). Dryland strength training vs. electrical stimulation in sprint swimming performance. The Journal of Strength \& Conditioning Research, 26(2), 497-505.

Gonzáles-Badillo, J.J., y Gorostiaga (1995). Fundamentos del entrenamiento de la fuerza: Aplicación al alto rendimiento deportivo. Barcelona: Inde.

Guilherme, L., Guglielmo, A., y Denadai, B. S. (2000). Assessment of anaerobic power of swimmers: the correlation of laboratory tests on an arm ergometer with field tests in a swimming pool. The Journal of Strength \& Conditioning Research, 14(4), 395398.

Hawley, J. A., y Williams, M. M. (1991). Relationship between upper body anaerobic power and freestyle swimming performance. International Journal of Sports Medicine, 12(01), 1-5.

Holfelder, B., Brown, N., y Bubeck, D. (2013). The influence of sex, stroke and distance on the lactate characteristics in high performance swimming. PloS one, $8(10)$, e77185.

Issurin, V. B., Kaufman, L. E., y Tenenbaum, G. (2001). Modeling of velocity regimens for anaerobic and aerobic power exercises in high-performance swimmers. The Journal of sports medicine and physical fitness, 41(4), 433-440. 
Johnson, R. E., Sharp, R. L., y Hedrick, C. E. (1993). Relationship of swimming power and dryland power to sprint freestyle performance: a multiple regression approach. Journal of Swimming Research, 9, 10-14.

Jorgić, B., Puletić, M., Okičić, T., y Meškovska, N. (2011). Importance of maximal oxygen consumption during swimming. Facta universitatis-series: Physical Education and Sport, 9(2), 183-191.

Klika, R. J., y Thorland, W. G. (1994). Physiological determinants of sprint swimming performance in children and young adults. Pediatric exercise science, 6, 59-59.

Lätt, E., Jürimäe, J., Mäestu, J., Purge, P., Rämson, R., Haljaste, K., y Jürimäe, T. (2010). Physiological, biomechanical and anthropometrical predictors of sprint swimming performance in adolescent swimmers. Journal of sports science \& medicine, 9(3), 398.

Lieber, R.L. y Friden, J. (2000). Functional and clinical significance of skeletal muscle architecture. Muscle nerve, 23(11): 1647-1666 Monu, J. M. (2013). Sport-Specific Training for a Competitive Freestyle Sprint Swimmer. Strength \& Conditioning Journal, 35(5), 48-55.

Nasirzade, A., Ehsanbakhsh, A., Argavani, H., Sobhkhiz, A., y Aliakbari, M. (2014, In press). Selected anthropometrical, muscular architecture, and biomechanical variables as predictors of 50-m performance of front crawl swimming in young male swimmers. Science \& Sports.

Newton, R. U., Jones, J., Kraemer, W. J., y Wardle, H. (2002). Strength and power training of Australian Olympic swimmers. Strength \& Conditioning Journal, 24(3), 7-15.

Ogita, F. (2006) Energetics in competitive swimming and its application for training. En: Biomechanics and medicine in swimming, X. Porto, 117-121.

Özçaldiran, B., y Durmaz, B. (2008). Isokinetic muscle strength for ankle extensors and flexors: a comparison between elite sprint runners and swimmers. The Journal of Sports Medicine and Physical Fitness, 48(3), 300-304.

Pelot, T., y Darmiento, A. (2012). Strength and Power Training for the Elite Swimmer: Can Weights Positively Impact Elite Swim Performance when "Elite Performance" Requires 15 - 25 Hours/Week of Practice? Olympic coach magazine, 23(2), 22-31.

Peyrebrune, M. C., Toubekis, A. G., Lakomy, H. K. A., y Nevill, M. E. (2012). Estimating the energy contribution during single and repeated sprint swimming. Scandinavian Journal of Medicine \& Science In Sports.

Rama, L., Santos, J., Gomes, P., y Alves, F. (2006). Determinant factors related to performance in young swimmers. BIOMECHANICS AND MEDICINE IN SWIMMING, X. Porto, 246-249.

Rebutini, V. Z., Pereira, G., Bohrer, R. C., Ugrinowitsch, C., Rodacki, A. L., de Maria, R. C., y Botânico (2014). Plyometric long jump training with progressive loading improves kinetic and kinematic swimming start parameters. Journal of Strength and Conditioning Research Publish Ahead of Print.

Ring, S., Mader, A., Wirtz, W., \& Wilke, K. (1996). Energy metabolism during sprint swimming. Biomechanics and medicine in swimming VII. E \& FN Spon, London, 177-184.

Rodriguez, F., y Mader, A. (2003). Energy metabolism during 400 and 100-m crawl swimming: computer simulation based on free swimming measurement. Biomechanics and Medicine in Swimming, IX, 373-378.

Ruzbarsky, P., y Turek (2006). Diagnosing of performance by the application of swimming tests. Biomechanics and Medicine in Swimming, X. Porto, 170-172. 
Salo, D., y Reiwald, S.A. (2008) Preparación física completa para la natación. Madrid: Tutor.

Seifert, L., Komar, J., Leprêtre, P. M., Lemaitre, F., Chavallard, F., Alberty, M., y Hellard, P. (2010). Swim specialty affects energy cost and motor organization. International journal of sports medicine, 31(9), 624.

Sharp, R. L., Troup, J. P., y Costill, D. L. (1982). Relationship between power and sprint freestyle swimming. Medine and Science in Sports and Exercises, 14(1), 53 56.

Siders, W. A., Lukaski, H. C., y Bolonchuk, W. W. (1993). Relationships among swimming performance, body composition and somatotype in competitive collegiate swimmers. The Journal of sports medicine and physical fitness, 33(2), 166-171.

Smith, D. J., Norris, S. R., y Hogg, J. M. (2002). Performance evaluation of swimmers. Sports Medicine, 32(9), 539-554.

Smolka, L., y Ochmann, B. (2013). A Novel Method of Anaerobic Performance Assessment in Swimming. The Journal of Strength \& Conditioning Research, 27(2), 533-539.

Stager, J. M., y Babington, P. (1995). Somatic Traits in Selection of Potential Elite Swimmers. In Athens: In Proceedings book of XI Fina world sports medicine congres. Stirn, I., Jarm, T., Kapus, V., y Strojnik, V. (2011). Evaluation of muscle fatigue during 100-m front crawl. European journal of applied physiology, 111(1), 101-113.

Soares, S., Machado, L., Lima, A., Santos, I., Fernandes, R., Correia, M., y Vilas-Boas, J. P. (2006). Velocimetric characterization of a $30 \mathrm{sec}$ maximal test in swimming: consequences for bioenergetical evaluation. In Xth International Symposium Biomechanics and Medicine in Swimming.

Strzala, M., y Tyka, A. (2009). Physical endurance, somatic indices and swimming technique parameters as determinants of front crawl swimming speed at short distances in young swimmers. Medicina Sportiva, 13(2), 99-107.

Toussaint, H. M., y Hollander, A. P. (1994). Energetics of competitive swimming. Sports Medicine, 18(6), 384-405.

Toussaint, H. M., Van den Berg, C, y Beek, W. J. (2002). "Pumped-up propulsion" during front crawl swimming. Medicine and science in sports and exercise, 34(2), 314-319.

Trinidad, A., y Lorenzo, A. (2012). Análisis de los indicadores de rendimiento en las finales europeas de natación en pruebas cortas y en estilo libre. Apunts: Educación física y deportes, (107), 97-107.

Vandewalle, H., Peres, G., Sourabie, B., Stouvenel, O., y Monod, H. (1989). Forcevelocity relationship and maximal anaerobic power during cranking exercise in young swimmers. International Journal of Sports Medicine, 10(6), 439-45.

Vasconcelos, A. (2000). Planificación y organización del entrenamiento deportivo (Vol. 24). Barcelona: Paidotribo.

West, D. J., Owen, N. J., Cunningham, D. J., Cook, C. J., y Kilduff, L. P. (2011). Strength and power predictors of swimming starts in international sprint swimmers. The Journal of Strength \& Conditioning Research, 25(4), 950-955.

Willems, T. M., Cornelis, J. A., De Deurwaerder, L. E., Roelandt, F., y De Mits, S. (2014). The effect of ankle muscle strength and flexibility on dolphin kick performance in competitive swimmers. Human movement science, 36, 167-176.

Zampagni, M. L., Casino, D., Benelli, P., Visani, A., Marcacci, M., y De Vito, G. (2008). Anthropometric and strength variables to predict freestyle performance 
times in elite master swimmers. The Journal of Strength \& Conditioning Research, 22(4), 1298-1307.

Zamparo, P., Capelli, C., Cautero, M., y Di Nino, A. (2000). Energy cost of front-crawl swimming at supra-maximal speeds and underwater torque in young swimmers. European journal of applied physiology, 83(6), 487-491. 